\title{
FLOWABLE PLATELET RICH FIBRIN A NEW TREND FOR ENHANCING BONE AUGMENTATION RESULTS IN MAXILLARY SINUS FLOOR AUGMENTATION. (RADIOGRAPHIC AND HISTOLOGIC EVALUATION)
}

\author{
Hesham S. Abdelmoneim* and Radwa T. Elsharkawy**
}

\begin{abstract}
Flowable (injectable) PRF became an increasingly favored approach for both soft and mineralized tissue healing due to the development of a three-dimensional fibrin network embedding within it; platelets, leukocyte cytokines, type I collagen, osteocalcin, and numerous growth factors. Flowable PRF enriched and vibrant bioactivity, simplified preparation, high healing potentials and easy incorporation with other biomaterials were the key aspects to stimulate the applications of flowable PRF in various medical fields. The current study aimed to evaluate if flowable PRF has superior bone augmentation properties over the conventional PRF in sinus floor augmentation procedures. In this study 18 maxillary sinuses were augmented, which were divided into 2 groups, group A maxillary sinuses were augmented using a mixture of xenograft bone substitute, group B maxillary sinuses were augmented using a mixture of xenograft bone substitute and flowable PRF.
\end{abstract}

Results: Clinically flowable PRF showed ease of preparation and improved the handling and manipulation of the bone graft particles. Radiographic results showed that Group A average gained bone height of $10.4 \pm 1.2 \mathrm{mms}$ while in group B mean gained bone height was $11.6 \pm 1.9 \mathrm{mms}$, bone densities results of both groups showed that bone density units of group A were $578 \pm 56$ Hounsfield Units(HU), while group B calculated augmented bone densities were $586.2 \pm 68 \mathrm{HU}$. Histologic evaluation stated that group I and group II revealed a lot of similarities regarding the amount and the quality of the newly formed bone.

There by concluding that flowable PRF resulted in similar results to that of the conventional PRF, however the ease of preparation and the ease of application and manipulation emphasizes its benefits and recommends its application in the implant and bone defects grafting scenarios.

KEY WORDS : Flowable PRF, injectable PRF, sinus lifting, dental implants, PRF in maxillary sinus

* Lecturer of Oral and Maxillofacial Surgery, Faculty of Dentistry, Cairo University.

** Lecturer of Oral Biology, Faculty of Dentistry, Future University in Egypt. 


\section{INTRODUCTION}

In implantology and surgical dentistry, efforts are continuously carried out to enhance wound healing process and new bone formation. Platelet rich fibrin (PRF) an autologous biomaterial rich in growth factors was a new step in the platelet gel therapeutic concept which was developed in France by Choukroun et al 2001. Since then efforts were fully targeting how to gain maximum benefits, utilize the PRF full potential and to improve its outcomes. PRF extraction attempts to accumulate platelets and cytokines in a matrix of autologous fibrin, in which leukocyte cytokines and platelet are embedded in large quantities. These cytokines intrinsically incorporated within the fibrin mesh that allows for their progressive efflux over 7-11 days as the fibrin meshwork disintegrates..$^{(1,4,5)}$

Also it could be considered as fibrin network of nano-scale fibers that can act as a scaffold for cell differentiation, migration and proliferation. Alternatively it could be thought of as a drug delivery system of growth factors, which accelerate healing and induce neo-angiogenesis. ${ }^{(1,6)}$

It has been previously postulated that stem cells reside in a unique microenvironment composed of an extracellular meshwork and resident cells referred to as stem cell niche, such vibrant microenvironments were also found in PRF. The positive results of PRF could be due to the more physiologically natural and progressive polymerization during centrifugation. ${ }^{(7)}$

PRF is a suitable scaffold for all bone tissue engineering utilizations because of its' microenvironment which is favorable for differentiation and proliferation of the osteo-competent cells (osteoblasts), thus PRF has the capability to act as a graft substitute with both osteo-inductive and osteo-conductive potentials. ${ }^{(8)}$

It has been noted that PRF glue provided mechanical support for the particulate bone graft, also that glue holds particulate bone tightly in a favorable configuration through cross-linking between the fibrin fibers which in turn mechanically stabilizes the architecture of the fibrin-based scaffold while maintaining a proper elastic-mechanical and biological behavior. In addition to its physical benefits it also accelerates the healing process by platelets incorporation into the fibrin glue from which numerous growth factors are effused upon their activation with thrombin. Thereby platelet rich fibrin has been heavily considered for augmentation of defected alveolar ridges. ${ }^{(9,10)}$

Flowable or what's also known as injectable platelet-rich fibrin (i-PRF) was developed in 2014 though modifying spin centrifugation parameters. Flowable PRF was gained when blood centrifuged at lower rotations per minute in a non-glass centrifugation tube. ${ }^{(7)}$

As handling of bone grafts was found to be quite challenging when dealing with extensive bony defects, the gluing property gained from the mixing of the graft material with flowable PRF was found to be of a great benefit. As the liquid fibrinogen in the flowable PRF slowly converts into fibrin that acts as an autologous fibrin binder. This strategy has been used by surgeons recently to create an agglomeration and or biomaterials coating to promote better wound healing potentials..$^{(13,4)}$

Previous Studies have monitored the efflux of various growth factors from flowable PRF, in accordance with its influence on different cells behavior as the fibroblasts and the osteoblasts. thereby emphasizing on the beneficial role of flowable-PRF in the healing process of both mineralized and soft tissues. Although, few studies currently described the cell content, morphology and growth factors in flowable PRF. The simplified preparation, and its easy association with other biomaterials were key aspects that promoted its use in various fields of dentistry as periodontics, bone augmentation procedures and implantology. ${ }^{(1,7,8,9)}$ 
Several sinus floor augmentation procedures have been introduced since the 1980s to create bone foundation that allows dental implants placement in the atrophied posterior maxilla. The created space has been filled with various graft materials to promote and guarantee new bone formation. Numerous graft materials have been applied as autografts, allografts, xenografts, alloplastic bone substitutes, platelet rich plasma (PRP), PRF and even any combinations. Despite autogenous bone being the gold standard but it results in donor site morbidity, thereby autogenous bone is not widely used in clinical practice and not favored by patients. Other graft materials also have their limitations, among which are risk of infection, insufficient amount, and elevated overall cost. Thus it has been documented that no graft material was superior over the others. ${ }^{(15,16)}$

PRF mixed bone substitutes or PRF alone has been used as a graft material for sinus augmentation procedures via both the lateral and crestal approaches. Promising results both clinical and radiological were documented stating that PRF had a good influence regarding new bone formation. ${ }^{(17)}$ There by the aim of this study was to evaluate if flowable PRF mixture with xenograft particulates resulted in better bone augmentation results (radiographic and histologic) over the conventional PRF in sinus floor augmentation procedures.

\section{MATERIALS AND METHODS}

\section{Trial design}

This was a double blinded, parallel grouped randomized controlled clinical trial. With allocation ratio of 1:1. Patients of this study were allocated into 2 groups. In control group (A), maxillary sinuses floors were augmented using a mixture of xenograft bone substitute (Tutogen Xenogenic bone 0.25-1 mm particles, Tutogen Medical. GmbH, Neunkirchen, Germany) and shredded PRF plug. While in the intervention group (B), maxillary sinuses were augmented using a mixture of xenograft bone substitute and flowable PRF.

\section{Participants}

This study was conducted on 10 male patients in whom 18 maxillary sinuses were augmented, selected from the out-patient clinic, Department of Oral and Maxillofacial Surgery, Faculty of Dentistry, Cairo University from February 2016 to November 2018. Simple randomization was performed using standardized paper sheets (group A and group B) placed in sealed opaque envelops. After accepting to be enrolled in the study, cases were assigned randomly to either group.

\section{Selection criteria were;}

- Patients should be free from any systemic condition that might affect the outcomes, or even contraindicate the surgical intervention.

- Edentulous posterior maxillary region with no history of previous attempts for grafting of the deficient alveolar ridge.

- Vertical alveolar bone height less than $4 \mathrm{mms}$.

- Sufficient width of the alveolar bone area (more than $5 \mathrm{mms}$ )

\section{Intervention}

All maxillary sinus floor augmentations in our study were performed via lateral open sinus approach, where a crestal and single anterior releasing incision was used to expose the lateral wall of the maxillary sinus, then sinus burs were used under copious amount of irrigation to open a window in the lateral maxillary wall relative to the site of desired bone augmentation. Sinus elevators (MSC, Korea) were used to reflect the schneidarian membrane (fig.1a), after the complete elevation the graft was then prepared. In group A PRF was prepared where $20 \mathrm{ml}$ of blood were withdrawn from the cubital vein of the patient, the withdrawn blood was then divided into two $10 \mathrm{ml}$ vaccutainers (plain tubes) and centrifuged for $15 \mathrm{~min}$ at 3500 rpm to obtain the PRF plug (Centra 4600, China) (Fig 2). Then the obtained plugs were fragmented 
and mixed with the bone graft particles to form the grafting mixture. In group B flowable or injectable PRF was obtained by same blood withdrawal technique but then centrifuged for 2 minutes at $3300 \mathrm{rpm}{ }^{(18)}$, the obtained Flowable PRF was then mixed with the xenograft bone chips and left to clot (settle) for 5 minutes where an aggregate of the flowable PRF and the xenograft was formed (Fig 3). Then the graft mixture was packed into the sinus to fully fill the created gap and support the elevated sinus membrane, then a collagen membrane (Tutgen medical Gmbh, Germany) was placed over the bony window followed by flap closure. Patients of both groups were instructed to administrate post-operative prophylactic antibiotics twice daily (Augmentin 1gm or amoxicillin and clavulanate potassium), analgesics 3 times per day (Brufen $600 \mathrm{mg}$ or IBUPROFEN) for 5 days together with

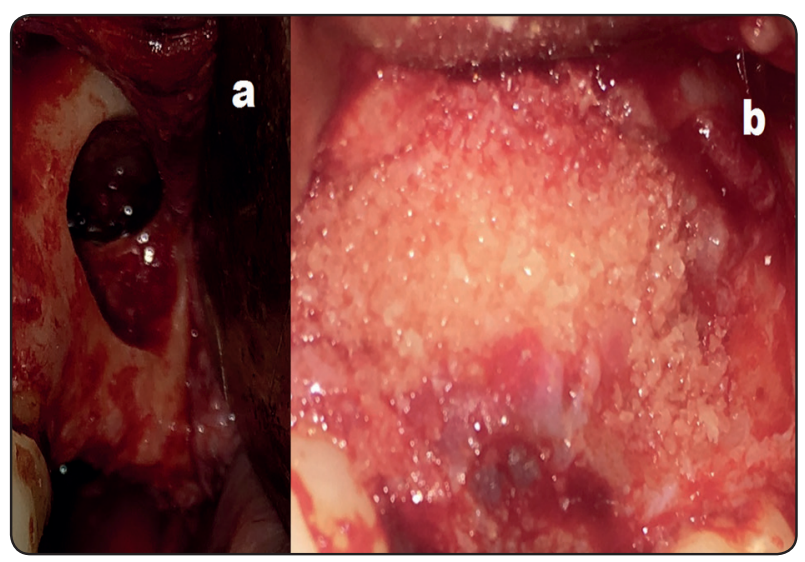

Fig. 1a; showing the lateral window with the reflected sinus membrane. 1b; showing the packed sinus with particulate graft mixture with flowable PRF. an anti-inflammatory injection of dexamethasone $80 \mathrm{mg}$ (immediately post-operative and $12 \mathrm{hrs}$ later). Oral hygiene measures were instructed to the patient (regular tooth brushing and use of chlorohexidine mouth wash 3 times daily). Patients were instructed to avoid all actions that could cause sinus pressure rise by mouth opening during coughing and sneezing, no straining, only gentle nose blowing and prohibited from any physical exercise for a week. All patients of both groups were recalled after 1 week for sutures removal.

Patients were recalled after 1 week, 2 weeks, 1 month and 4 months post operative for the clinical and radiographic evaluations.

Radiographic assessment for both groups was performed by a series of Cone Beam CTs (3D On Demand) taken immediately post-operative and

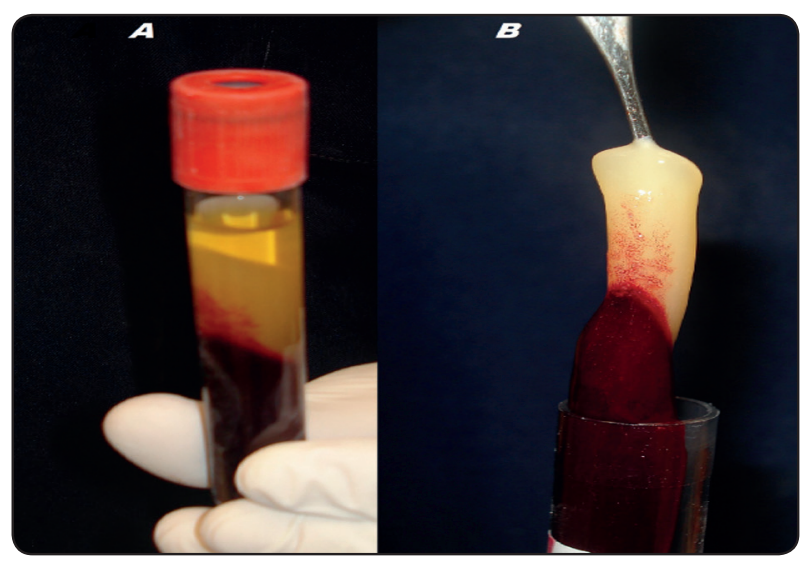

Fig. 2: A; showing the obtained flowable PRF and B; showing the obtained PRF plug.

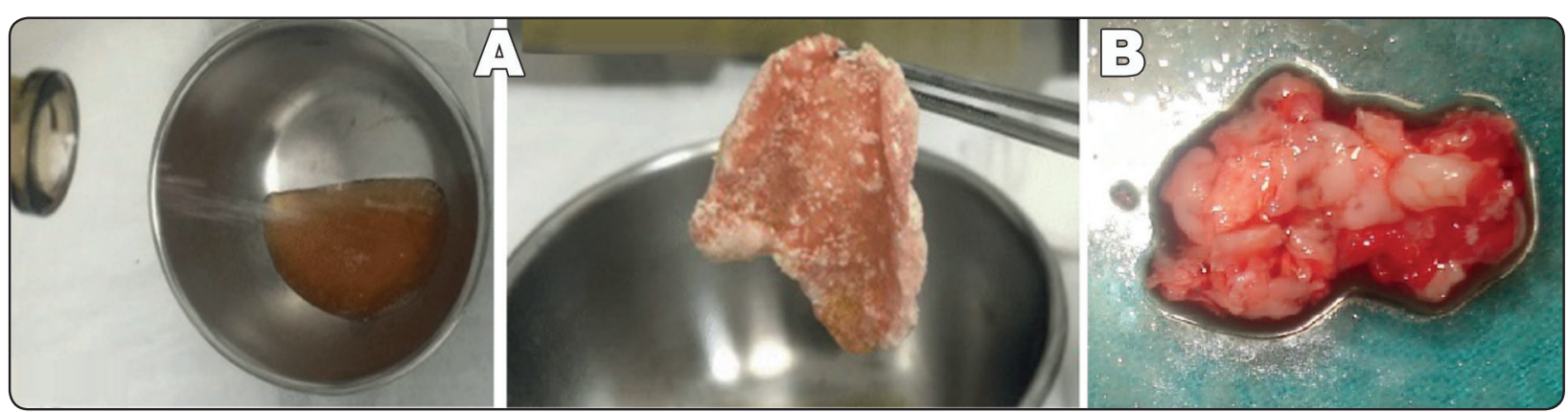

Fig. 3: A; showing the graft addition to the flowable PRF(group B) and the formed aggregate. B; showing the mixture of fragmented PRF and the bone graft (group A). 
after 4 months to assess the vertical gain in sinus floor elevation and the final grafting density.

Histological evaluation was done on core biopsies taken from the site of dental implant osteotomy via a $3 \mathrm{~mm}$ external diameter trephine bur after 4 months of the sinus floor grafting. The biopsy specimens were fixed immediately in $10 \%$ neutral buffered formalin. After complete decalcification, specimens were processed, infiltrated in paraffin wax and embedded in the center of wax blocks. The embedded specimens were cut into $5 \mu \mathrm{m}$ thick sections. Specimens were sectioned and were stained by Hematoxylin and Eosin (H\&E) stain. After fixation, specimens were washed properly under running water, dehydrated by transferring through ascending concentrations of alcohol. The sections were stained by Hematoxylin and Eosin (H\&E) stain to be examined by light microscope (Trinocular microscope Olympus, BX46).

Statistical analysis was performed via Microsoft excel statistical analyzer, AVERAGE, STDEV and $P$ value calculated by $T$ test to compare variables between the two groups. The results were considered statistically significant if the $\mathrm{p}$ value was $\leq 0.05$.

\section{RESULTS}

Clinical follow up revealed that all our cases didn't suffer from any post operative complications (dehiscence / infection). All patients reached our 4 months post operative evaluation mark point, and core biopsies were collected for histological analysis at the time of dental implants installations.

\section{Radiographic results}

Post operative CBCTs of both groups were assessed to obtain gained alveolar bone height and radiographic bone densities (fig.4). Group A showed an average gained bone height of $10.4 \pm 1.2$ mms while in group B mean gained bone height was $11.6 \pm 1.9 \mathrm{mms}$ (chart 1 ) and a calculated $\mathrm{P}$ value of 0.53 with a statistical significance mark point of $\leq 0.05$. Bone densities results of both groups showed that bone density units of group A were $578 \pm 56$
Hounsfield Units(HU), while group B calculated augmented bone densities were $586.2 \pm 68 \mathrm{HU}$ (chart 2) and with calculated p value of 0.68 with a statistical significance mark point of $\leq 0.05$.

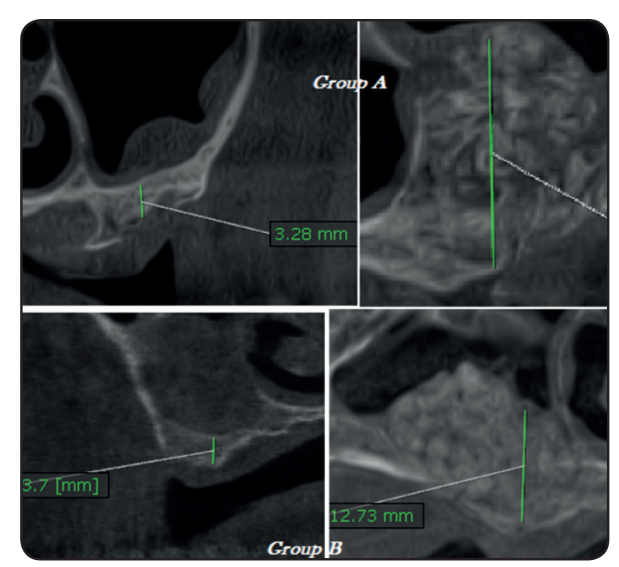

Fig. (4) Showing a sample of radiographic results taken of group $\mathrm{A}$ and $\mathrm{B}$.

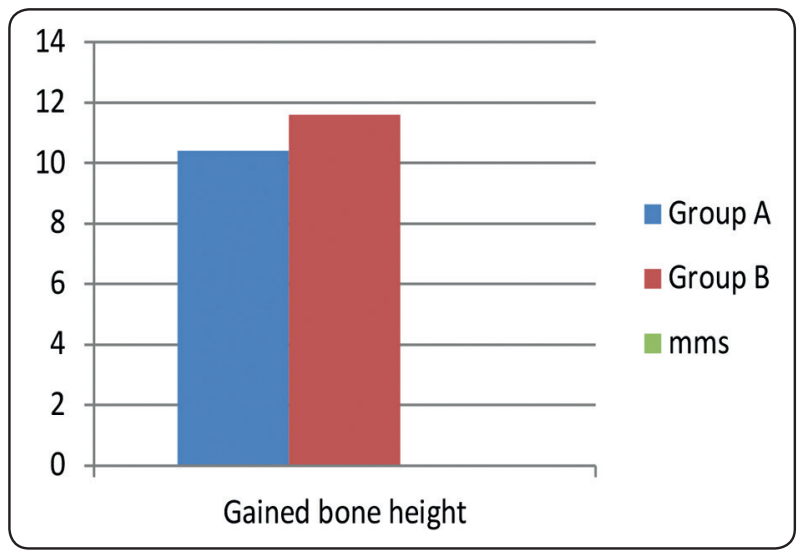

Chart 1; Showing gained alveolar bone height after 4 months of sinus floor grafting of group A and B.

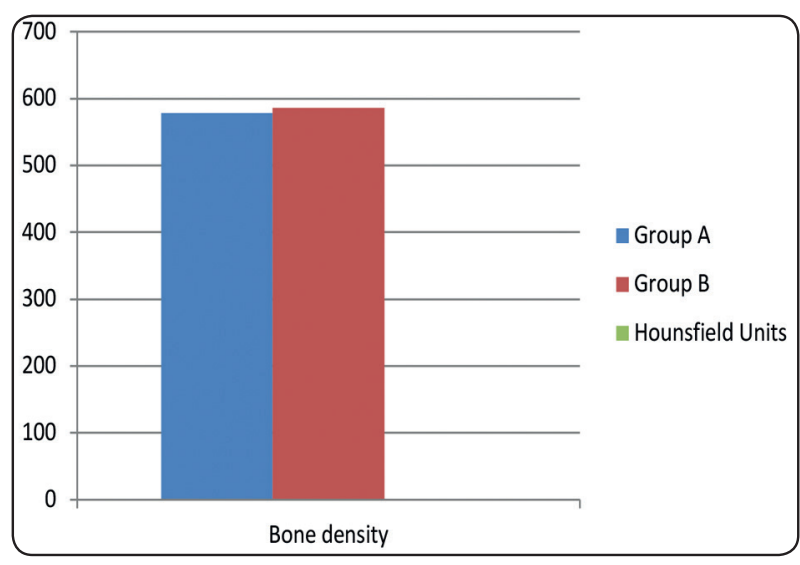

Chart 2; Showing bone densities of group A and B. 


\section{Histological results}

\section{Microscopic assessments Hematoxylin and Eosin (H\&E) stain}

The histological assessments of the specimens after 4 months healing period showed evidence of new bone formation in the two maxillary augmented groups. The histological pictures of group A and group B revealed a lot of similarities regarding the amount and the quality of the newly formed bone.

The specimens showed islets of the newly formed bone trabeculae surrounding highly vascularized bone marrow which are filled by fibro-cellular connective tissue rich in fibroblasts, undifferentiated mesenchymal cells and inflammatory cells.

The new bone trabeculations are lined by osteoblasts and the osteocytes on the boundaries appeared irregularly arranged while the osteocytes at the center tend to be more organized in the circular pattern of the Haversian system. Basophilic lines of demarcation of bone remodeling as well as resting lines were observed. Remnants of the graft materials were observed accompanying the newly formed bone; they were easily identified because of their staining. No signs of inflammatory cells of fibrotic tissues were observed.

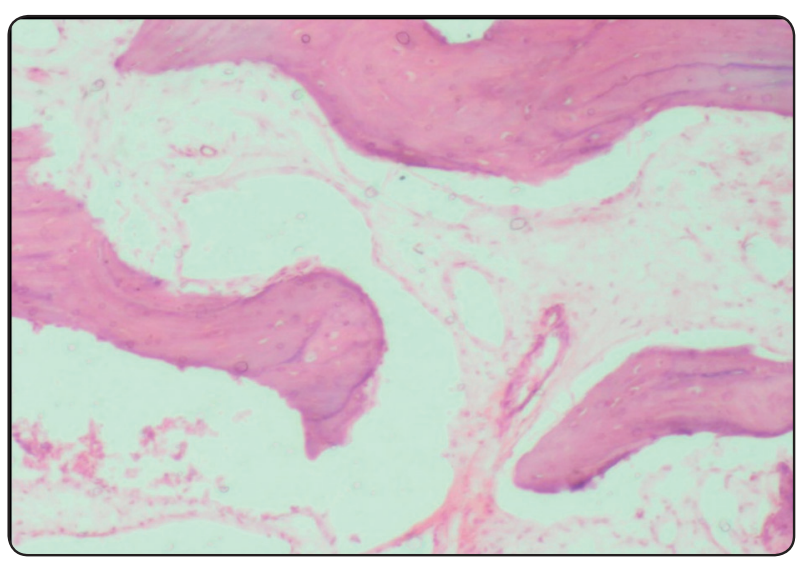

Fig. (5) Photomicrograph of the group A specimens showing interconnected bone trabeculae surrounding highly vascularized bone marrow, remnants of the graft materials. (H\&E x40)

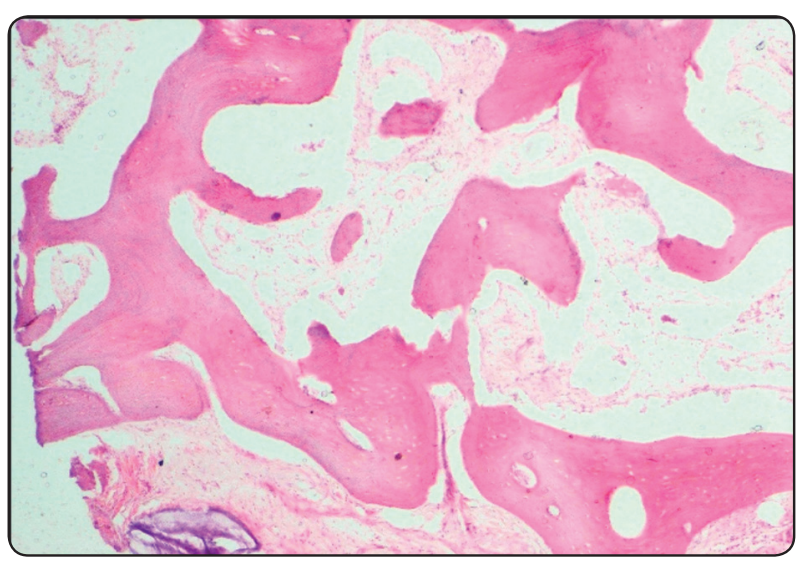

Fig. (6) Photomicrograph of the group A specimens showing the newly formed bone trabeculae lined by osteoblasts ,bone marrow spaces filled by filled by fibro-cellular connective tissue rich in fibroblasts, blood vessels, undifferentiated mesenchymal cells and inflammatory cells. (H\&E x100)

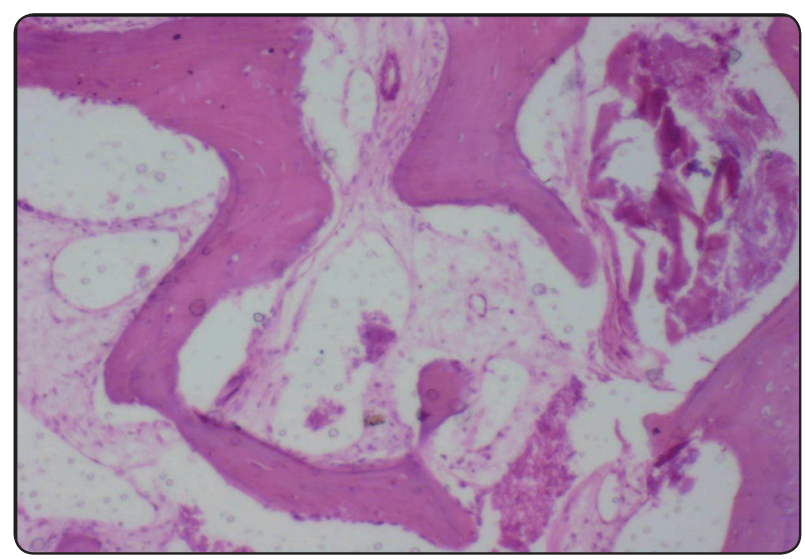

Fig. (7) Photomicrograph of the group B specimens showing connected islands of new bone trabeculae, basophilic line of demarcations and highly vascularized bone marrow. (H\&E x40

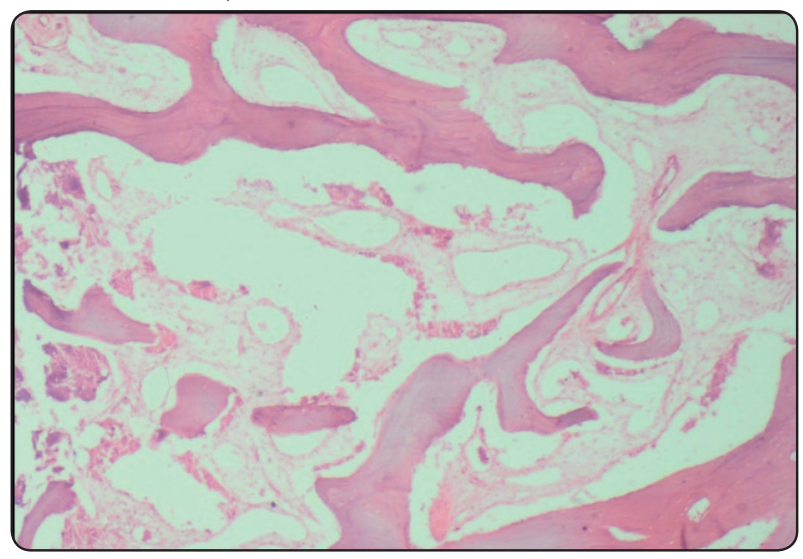

Fig. (8) Photomicrograph of the group B specimens showing basophilic line of demarcations representing bone remodeling ( $\mathrm{H} \& \mathrm{E} \mathrm{x} 100)$ 


\section{DISCUSSION}

This study was conducted aiming to determine if flowable PRF was an improvement or even added benefits over the thoroughly researched PRF. Evaluating the radiographic results of the current study showed that average gained bone height of $10.4 \pm 1.2 \mathrm{mms}$ in the sinuses augmented using PRF with xenograft while the mean gained bone height was $11.6 \pm 1.9 \mathrm{mms}$ in the sinuses grafted using flowable PRF Xenograft mixture and the calculated $\mathrm{P}$ value for that comparison was 0.53 which stated that the obtained results were statistically insignificant. The calculated radiographic bone densities of the augmented sinuses of both groups showed that the mean bone density of group A was $578 \pm 56$ Hounsfield Units (HU), while group B calculated augmented bone densities were 586.2 $\pm 68 \mathrm{HU}$ and with calculated p value of 0.68 with statistical significance mark point of $\leq 0.05$, thus denoting statistically non-significant results.

There have been several publications of sinus floor augmentations using PRF as an adjunctive grafting material and promising survival rates were reported which denoted that PRF was a reliable sole graft material that could be used in grafting procedures and implant dentistry. ${ }^{(11,13)}$ Choukroun et al, mixed PRF with a freeze-dried bone allograft and stated that the mixture reduced and improved healing time. Also is has been described that increased neo- bone formations in histological evaluation of PRF mixture with xenograft were evident. ${ }^{(19)}$

In previous studies utilizing a combination of bone graft materials and PRF, conclusions were stated that alveolar ridge augmentation using bone grafts and PRF glue with simultaneous dental implants placements might effectively enhance vertical bone alveolar ridge height and enhance dental implants osseointegration. Also stating that PRF does not appear to enhance cellular proliferation on the long term, but however plays an important role in graft revascularization by supporting angiogenesis. ${ }^{(9)}$
Although initially in liquid phase, macroscopic findings demonstrated that flowable PRF initiates a fibrin polymerization process by acquiring a gel phase over time. Histological findings showed a uniform leukocyte (mainly lymphocytes) and platelet distribution throughout the test specimens. In literature, histological analyses on PRF revealed non-uniform distribution of these cell groups in the agglomerates. ${ }^{(1)}$ It has been stated that flowable PRF was able to undergo blood hemolysis while maintaining its morphological aspects and properties over a period of 10 days.and thereby flowable PRF formed a dynamic fibrin gel that acted as a scaffold for wound healing. ${ }^{(20,21)}$

That three-dimensional fibrin matrix also played a key role in tissue repair although most studies have primarily focused on growth factors and their effect. Fibrin acts as a scaffolding biological material for agglomeration of adherent cells (platelets and leucocytes) at the site of tissue healing. In addition, fibrin acts as a carrier of growth factors in a wellcontrolled release system that sustains proper bioactivity over the healing period. ${ }^{(12,22)}$

Several histological and morphological analysis documented that flowable PRF provided a threedimensional fibrin network that embedded platelets, leukocytes, type I collagen, osteocalcin, and growth factors. The physicochemical characterization of flowable or injectable PRF remains an important step forward to the understanding of their clinical effects and could provide important guidelines for the different tissue and/or injury conditions that imply its use in the future. And thereby recommending that combinations of flowable PRF and other synthetic biomaterials should be further studied to fully reveal its potential. ${ }^{(1)}$

From our study it has been clear that the use of the flowable PRF made the xenograft more controllable and easier to manipulate during the grafting procedure due to the aggregate the resulted from the fibrin clot that developed after leaving the 
flowable PRF to settle for a while. In addition the graft particles were not dispersed all over the sinus window and cavity, didn't escape under the flap, and were easier to place and pack in the sinus cavity created by reflecting the sinus lining membrane. All our postulations were based on clinical practice during the grafting procedures of that group, while all our augmented sinuses received dental implants after 4 months and as all our implants successfully integrated and were loaded after 4-5 months after dental implants installations, however the exact benefit of using the flowable PRF technique over the PRF couldn't be felt clinically or even radiographically and thereby was left to the histological analysis of the core biopsies taken at the time of dental implants installation from both of our groups to determine if our hypothesis was correct or even plausible. Histological evaluation revealed evidence of new bone formation in the two maxillary augmented sinus groups with similarities regarding the amount and the quality of the newly formed bone.

\section{CONCLUSION}

- Flowable platelet rich fibrin could be used as an adjunctive graft material in sinus floor augmentation procedures due to increased ease of preparation, application and graft manipulation over the conventional PRF.

- Flowable PRF isn't superior to conventional PRF in new bone formation quality both radiographic and histologically.

\section{REFERENCES}

1. Varela.H, Souza.J, Nascimento.R, Júnior.R, Vasconcelos.R, Cavalcante.R, Guedes.P and Araújo.A. Injectable platelet rich fibrin: cell content, morphological, and protein characterization. Clinical Oral Investigations. Mar; 23(3):1309$1318,2019$.

2. Dohan D.M., Choukroun J., Diss A., Dohan S.L., Dohan A.J., Mouhyi J. and Gogly B.: Platelet-rich fibrin (PRF): a second-generation platelet concentrate. Part I: technological concepts and evolution. Oral Surg Oral Med Oral Pathol Oral Radiol Endod. 101; 37-44, 2006.

3. Galing V.L.W., Açil Y., Springer I.N., Hubert N. and Wiltfang J.: Platelet-rich Plasma and Platelet-richfibrin in human cell culture. Oral Surg Oral MedOral Pathol Oral Radiol Endod. 108; 48-55, 2009.

4. Wu C-L, Lee S-S, Tsai C-H, Lu K-H, Zhao J-H, Chang Y-C. Platelet-rich fibrin increases cell attachment, proliferation and collagen-related protein expression of human osteoblasts. Aust Dent J. 57(2):207-212, 2012.

5. Castro AB, Meschi N, Temmerman A, et al. Regenerative potential of leucocyte and platelet-rich fibrin. Part B: sinus floor elevation, alveolar ridge preservation and implant therapy. A systematic review. J Clin Periodontol. 44(2):225- 234, 2017.

6. Del Corso M, Dohan Ehrenfest DM. Immediate implantation and peri-implant Natural Bone Regeneration (NBR) in the severely resorbed posterior mandible using Leukocyte-and Platelet-Rich Fibrin (L-PRF): a 4-year follow up. http://asnanportal.com/images/dental_report/periodonticsoral surgeryimplants/poseido-201312109-16-delcor-copia. pdf. 2013.

7. Dawson E., Mapili G., Erickson K., Taqvi S. and Roy K.: Biomaterials for stem cell differentiation. Advanced Drug Delivery Reviews. 60; 215-228, 2008.

8. Gassling V., Douglas T/, Warnke P.H., Acil Y., Wiltfang J. and Becker S.T.: Platelet-rich fibrin membranes as scaffolds for periosteal tissue engineering. Clin. Oral Impl. Res. 21; 543-549, 2010.

9. Hyeon Jung Lee, Byung Ho Choi, Jae Hyung Jung, Shi Jiang Zhu, Seoung Ho Lee, Jin Young Huh, Tae Min You and Jingxu Li.: Vertical alveolar ridge augmentation using autogenous bone grafts and platelet-enriched fibrin glue with simultaneous implant placement. Oral Surg Oral Med Oral Pathol Oral Radiol Endod. 105; 27-31, 2008.

10. Thorn J.J., Sørensen H., Weis-Fogh U. and Andersen M.: Autologous fibrin glue with growth factors in reconstructive maxilla facial surgery. Int J Oral Maxillofac Surg. 33; 95-100, 2004.

11. Wang X, Zhang Y, Choukroun J, Ghanaati S, Miron RJ. Effects of an injectable platelet-rich fibrin on osteoblast behavior and bone tissue formation in comparison to platelet-rich plasma.Platelets. doi:10.1080/09537104.2017.1293807.18. 2017 . 
12. Kobayashi M, Kawase T, Horimizu M, Okuda K, Wolff LF, Yoshie H. A proposed protocol for the standardized preparation of PRF membranes for clinical use. Biologicals. 40(5):323-329, 2012.

13. Wang X, Zhang Y, Choukroun J, Ghanaati S, Miron RJ. Behavior of gingival fibroblasts on titanium implant surfaces in combination with either injectable- PRF or PRP. Int J Mol Sci.18(2), 2017.

14. Miron RJ, Fujioka-Kobayashi M, Hernandez M, et al. Injectable platelet rich fibrin (i-PRF): opportunities in regenerative dentistry? Clinical Oral Investigations. 1-9, 2017.

15. Boyne P. J., James R. A. Grafting of the maxillary sinus floor with autogenous marrow and bone. Journal of Oral Surgery. 38(8):613-616, 1980.

16. Ali S., Bakry S. A., Abd-Elhakam H. Platelet-rich fibrin in maxillary sinus augmentation: a systematic review. Journal of Oral Implantology. doi: 10.1563/aaid-joi-d-14-00167. 41(6):746-753, 2015.

17. Toffler M., Toscano N., Holtzclaw D. Osteotome-mediated sinus floor elevation using only platelet-rich fibrin: an ear- ly report on 110 patients. Implant Dentistry. doi: 10.1097/ id.0b013e3181f57288. 19(5):447-456, 2010.

18. Mourão.C, Valiense.H, Melo.E, Mourão.N and Maia.M. Obtention of injectable platelets rich-fibrin (i-PRF) and its polymerization with bone graft: technical note. Revista do Colégio Brasileiro de Cirurgiões. 42. 421-423, 2015.

19. Choukroun J., Adda F., Schoeffler C. and Vervelle A.: Une opportunité en paro-implantologie: le PRF. Implantodontie. $42 ; 55-62,2001$.

20. Yajamanya SR, Chatterjee A, Babu CN, Karunanithi D. Fibrin network pattern changes of platelet-rich fibrin in young versus old age group of individuals: A cell block cytology study. J Indian Soc Periodontol. 20(2):151, 2016.

21. Dohan Ehrenfest DM, Pinto NR, Pereda A, et al. The impact of the centrifuge characteristics and centrifugation protocols on the cells, growth factors, and fibrin architecture of a leukocyte- and platelet-rich fibrin (L-PRF) clot and membrane. Platelets. 1-14, 2017.

22. Clark Raf. Fibrin and Wound Healing. Ann N Y Acad Sci. 936(1):355- 367, 2006. 